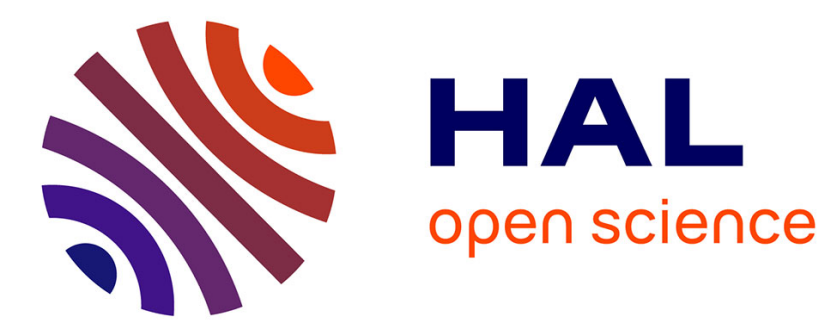

\title{
Entropy Coding for Networked Controlled Systems
}

Carlos Canudas de Wit, Jonathan Jaglin, Crisanto Vega

\section{To cite this version:}

Carlos Canudas de Wit, Jonathan Jaglin, Crisanto Vega. Entropy Coding for Networked Controlled Systems. ECC 2007 - European Control Conference, Jul 2007, Kos, Greece. pp.s/n. hal-00394991

\section{HAL Id: hal-00394991 https://hal.science/hal-00394991}

Submitted on 13 Jun 2009

HAL is a multi-disciplinary open access archive for the deposit and dissemination of scientific research documents, whether they are published or not. The documents may come from teaching and research institutions in France or abroad, or from public or private research centers.
L'archive ouverte pluridisciplinaire HAL, est destinée au dépôt et à la diffusion de documents scientifiques de niveau recherche, publiés ou non, émanant des établissements d'enseignement et de recherche français ou étrangers, des laboratoires publics ou privés. 


\title{
Entropy Coding for Networked Controlled Systems
}

\author{
Carlos Canudas-de-Wit, Jonathan Jaglin and Kelit Crisanto Vega \\ Departement d'Automatique de Grenoble, CNRS, GIPSA-Lab. \\ NeCS, INRIA-CNRS project-team, FRANCE. \\ carlos.canudas-de-witeinrialpes.fr, jonathan.jaglin@inpg.fr
}

\begin{abstract}
The aim of this paper is to explore yet another variant of the Delta-Modulation Coding structure to improve data transmission efficiency in the context of Networked Controlled Systems. High compression rates can only be reached by the use of entropy coding. Entropy coding assigns some probability distribution to the events. In that way, the mean code length can be improved. The paper studies several issues resulting from this type of coding design and assets the stability properties needed for this type of coding to operate properly.

Index Terms-Entropy coding, Networked controlled systems, NCS, quantized systems.
\end{abstract}

\section{INTRODUCTION}

$\mathbf{T}$ His paper deals with systems interconnected by a communication network where information is transmitted via a particular coding algorithm. Many of such type of control architectures have been studied in the past. Some examples are: [8], [4], [11], [12], [14], [10], [6], [1], among others.

In particular, delta modulation $(\Delta-M)$ has been used recently in this context [3] as a mean of reducing the number of transmitted bits while preserving a methodological and simple closed-form algorithm for the coding design. Delta modulation is a well-known differential coding technique used for reducing the data rate required for voice communication, see [13]. The standard technique is based on synchronizing a state predictor on emitter and receiver and just sending a one-bit error signal corresponding to the innovation of the sampled data with respect to the predictor. The prediction is then updated by adding a positive or negative quantity (determined by the bit that has been transmitted) of absolute value $\Delta$, a known parameter shared between emitter and receiver.

We have recently investigate the closed-loop properties of the $\Delta-M$ algorithm when used in the feedback loop. Our results in [3] have suggested some modification of the original form of the $\Delta-M$ algorithm to improve the closedloop properties when used in feedback within the context of Networked controlled systems (NCS). The results showed that the stability domain and the resulting precision of the $\Delta-M$ is limited by the position of the largest unstable pole of the system. Although this can be improved by increasing the sampling rate, or by the use of extra bits [7], both possibilities are clearly limited by the maximum permissible data transmission rate. Further studies, have also shown that it is possible to make the modulation gain adaptive so as to improve the global stability results [5].
The aim of this work is to explore yet another variant of the $\Delta-M$ structure to improve data transmission efficiency in the context of NCS. High compression rates can only be reached by the use of entropy coding. Entropy coding is a source coding that assigns some probability distribution to the events. In that way, the mean code length can be reduced, as it will be shown here.

A pre-requisite for the entropy coding strategy is to design a mechanism with the ability to quantify and to differentiate stand-still signal events, to changes in the source (level crossing detector, denoted here as $\varphi_{L D}$ ). For instance, this can be done by defining an alphabet where the source signal information is contained in the time interval between level crossing and in the direction of the level crossing. As suggested in [9], by assigning strings of the 2-tuple 00 to represent the time between signal level crossing, and 01 and 10 to denote the direction of level crossing, the output of the level crossing detector contains a high probability of the 0 symbol with makes it suitable for an entropy encoder to attain a "good" overall compression ratio. A fundamental difference with the classical $\Delta-M$ algorithm is that the error is coded on the basis of a 3-valued alphabet rather than a 2-valued one. The role of the entropy coding here is to render more efficient the transmission by improving in the mean number of bits per unit of time needed for the transmission.

The overall coding strategy studied here is composed of two main blocks: an event-based (EB) coding, and a variable length-block entropy (VLE)coding scheme. The overall scheme is shown in Figure 1. The paper aims at studding the closed-loop properties of such arrangement, and investigate the possible improvement in terms of compaction ration. Due to the fact that the VLE block is a distortion-less coding, and for simplicity reasons, the focus of this study is directed toward the study of the stability properties of the first coding block only.

\section{A. Definitions}

Signals are sampled on the basis of the time interval $T_{s}$, that is at $T_{s}, 2 T_{s}, \ldots k T_{s}$.

$r_{k}$ : reference signal,

$x_{k}$ : system output,

$\hat{x}_{k}$ : estimated(reconstructed) output,

$\tilde{x}_{k}:$ true estimated error, $\tilde{x}_{k}=x_{k}-\hat{x}_{k}$,

$\hat{\tilde{x}}_{k}$ : approximated estimated error, obtained after reconstruction, i.e. $\hat{\tilde{x}}_{k}=\left\{\varphi_{L D}^{-1} \circ \varphi_{L D}\right\}\left(\tilde{x}_{k}\right)$, with $\varphi_{L D}^{-1} \circ \varphi_{L D} \neq 1$. 


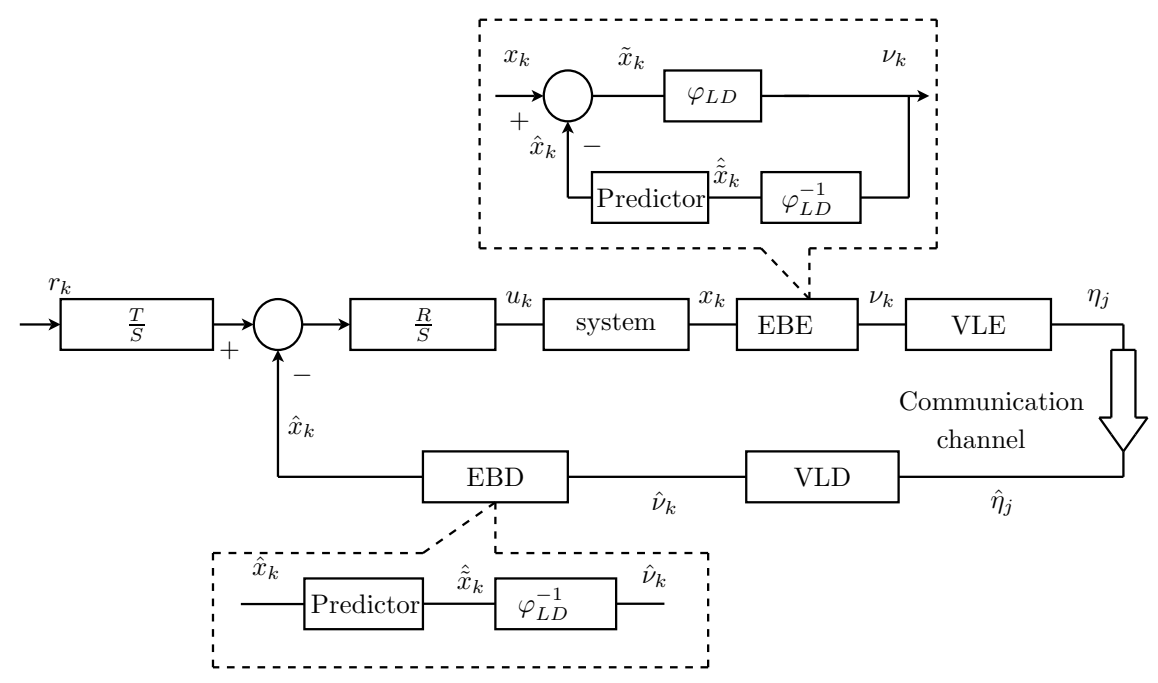

Fig. 1. Block diagram of the non uniform entropy coding in the feedback loop.

$\Delta:$ step interval used for level detection and to reconstruct $\hat{\tilde{x}}_{k}$,

$\delta_{k}: 3$-level valued integer signal: $\{-1,0,1\}$

$\nu_{k}:$ 2-bits binary signal

$\eta_{j}$ : variable length binary signal to be send by the channel (output of the VLE block) in asynchronous fashion, at the time instants multiples of $T_{s}$. The index $j$ captures this asynchronism.

$u_{k}$ : control input

\section{B. Assumptions}

The hypothesis used in the results presented in this paper, are the following:

- The transmitted information is binary

- Only encoder-to-decoder information transmission is allowed (feedback between decoder to encoder is forbidden),

- Reliable noiseless channel transmission is considered (no data lost, or information distortion, no transmission delays). See [2] for the treatment of transmission delay in this context.

\section{PROBLEM SET UP}

We consider the following SISO discrete-time linear system (possible unstable), of the form,

$$
x_{k}=\frac{B\left(q^{-1}\right)}{A\left(q^{-1}\right)} u_{k}
$$

together with a RST controller,

$$
u_{k}=\frac{R\left(q^{-1}\right)}{S\left(q^{-1}\right)}\left\{\frac{\gamma}{T\left(q^{-1}\right)} r_{k}-\hat{x}_{k}\right\}
$$

where $r_{k}$ is the reference, $\hat{x}_{k}$ is the estimated of the system output $x_{k}$, and $R\left(q^{-1}\right), S\left(q^{-1}\right), T\left(q^{-1}\right)$ are the control polynomials in the delay operator $q^{-1}$. They also satisfy:

$$
T=R B, \quad S A+R B=A_{c l}, \quad \gamma \triangleq A_{c l}(1)
$$

with $A_{c l}$ being the stable closed-loop polynomial, and $\gamma$ the static gain needed to reach unitary zero-frequency gain. For simplicity, we will omit the use of the argument $\left(q^{-1}\right)$ when not explicitly needed.

The coding process consists in: 1) encoding the system output $x_{k}$,2) transmitting the coding sequence through the communication channel, and 3) decoding the received information to produce the estimated $\hat{x}_{k}$. The complete sequence can be seen as estimation process.

a) Forced synchronization under asynchronous transmission: The time basis for the system and the controller description is defined with respect to $T_{s}$. However, due to the variable length characteristic inherent to the entropy coding, the transmission of the coded signal $\nu_{j}$ is then done asynchronously at multiples of $T_{s}$. For instance, for a choice of a VLE coding of length $N=3$, the coded signal can be sent either at; $T_{s}$, or $2 T_{s}$, or $3 T_{s}$ as shown in Table I. The index $j$ captures this asynchronism.

It is assumed here that when the receiver does not receive information (this may happen if the run sequence include a stand-still event for some $k$ ) the receiver hold the lastreceived value $\hat{\eta}_{k-1}$ until a new change of level is detected. By this mechanism, the signals at the receiver can be resynchronized to the time basis $T_{s}$. This is the reason why the control formulation is stated in a discrete-time synchronous representation with the sole index $k$, as described next.

b) Nominal closed-loop transfer function: Assume a perfect transmission process (i.e. $\hat{x}_{k} \equiv x_{k}$ ), then the control law (2) gives the following nominal closed-loop relation,

$$
x_{k}=\frac{\gamma}{A_{c l}\left(q^{-1}\right)} r_{k}
$$

c) Perturbed closed-loop transfer function: Consider the case of interest where information is transmitted by the channel and quantized, i.e. $\hat{x}_{k} \neq x_{k}$. Then, the error transfer function is 


$$
x_{k}=\frac{\gamma}{A_{c l}\left(q^{-1}\right)} r_{k}+W\left(q^{-1}\right) \tilde{x}_{k}
$$

where $\tilde{x}_{k}=x_{k}-\hat{x}_{k}$ is the estimation error, and $W=$ $B R / A_{c l}$. As $A_{c l}$ defines a stable polynomial, the output $x_{k}$ is kept bounded as long as $\tilde{x}_{k}$ is bounded as well.

The problem is then to design the coding process that defines the output $\hat{x}_{k}$ preserving closed-loop properties. This process is described next.

\section{Coding Process}

The coding (encoding/decoding) process is composed of several steps, described by the following operations:

$$
x_{k} \underbrace{\mapsto}_{E B E} \nu_{k} \underbrace{\mapsto}_{V L E} \eta_{k} \underbrace{\Rightarrow}_{\text {channel }} \hat{\eta}_{k} \underbrace{\mapsto}_{V L D} \hat{\nu}_{k} \underbrace{\mapsto}_{E B D} \hat{x}_{k}
$$

As shown in Figure 1, the encoder (respectively the inverse decoder) operation is composed of two separate blocks:

- The event-based encoder EBE (respectively, event-based decoder EBD). This block maps $x_{k} \mapsto \nu_{k}$ (respectively, the decoder maps $\hat{\nu}_{k} \mapsto \hat{x}_{k}$ ). This block includes a level detector $\varphi_{L D}$, and a model-based predictor, MBP, similar to the one proposed in [3], and

- A variable length entropy encoder VLE (respectively, variable length decoder VLD) mapping the binary signal $\nu_{k}$ (run sequence, see TableI) to the $N$-bits $\eta_{j}$ (respectively, the decoder maps $\hat{\eta}_{j} \mapsto \hat{\nu}_{k}$ ). This block includes the synchronization process described before.

\section{A. Description of the Event-based Coding EBC}

Elements composing the Event-based Coding EBC are the level detector $\varphi_{L D}$, and the model-based predictor.

1) The Level Detector: The operation principe of the level detector is shown in Figure 2. The signal detection levels are uniformly spaced by the quantum $\Delta$. The level detector device produces a signal (identified by ' $01^{\prime}$ or ' $10^{\prime}$ ) whenever a level crossing takes place, and a ' $00^{\prime}$ if the signal remains within the level. While two symbols are used to characterize the level changes, one more symbol can be used to quantize time intervals. Then 01 indicates upward crossing, 10 downward crossing, and 00 is used to code the timeinterval between crossing.

To illustrate this consider the example of Figure 2, see [9]. We assume that uniform samples are taken every time $T_{s}$, then $m$ samples are taken in the time interval $T_{i}=t_{i}-$ $t_{i-1}$ before a cross level takes place. As two levels (upward) crossing happen within this interval, the binary representation of this situation by the level crossing detector produce the following signal,

$$
01, \underbrace{00,00, \ldots 00}_{m-\text { pairs }}, 01
$$

This sequence has then high probability of 0 s, and thus suited for entropy coding.

To make this process operational, we introduce the operator $\varphi_{L D}: \tilde{x}_{k} \mapsto \nu_{k}$, which takes the error signal, $\tilde{x}_{k}$, and

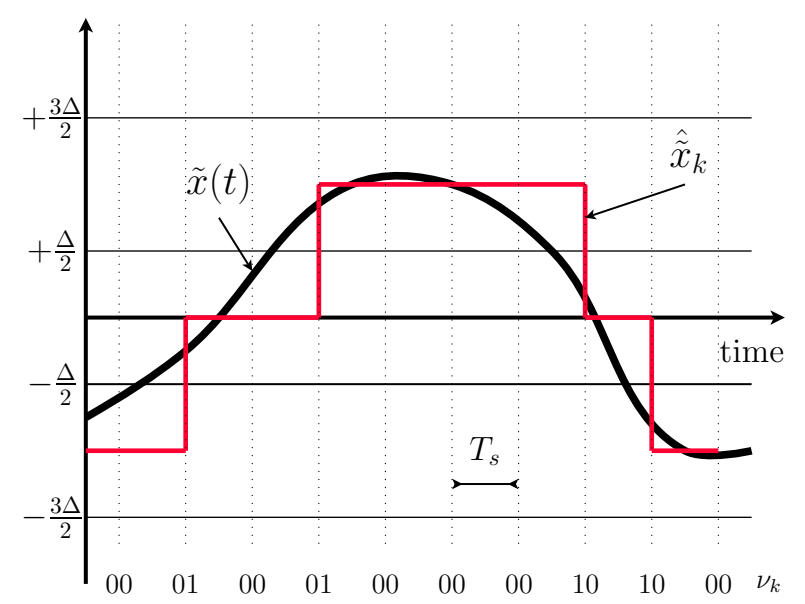

Fig. 2. Illustration of the level detector working operation principe.

codes the output $\nu_{k}$ into a 3 -valued one $\delta_{k} \in\{-1,0,1\}$. That is:

$$
\begin{aligned}
& l_{k}=\left\lfloor\frac{\tilde{x}_{k}}{\Delta}-\frac{1}{2}\right\rfloor \\
& \delta_{k}=\left\{\begin{array}{rr}
1 \text { if } & l_{k}>l_{k-1} ; \text { one level is crossed upwards } \\
0 \text { if } & l_{k}=l_{k-1} ; \text { signal stay at the actual level } \\
-1 \text { if } & l_{k}<l_{k-1} ; \text { one level is crossed downwards }
\end{array}\right.
\end{aligned}
$$

with $\Delta$ the level threshold and $\lfloor\cdot\rfloor$ the floor operator which rounds to the smaller integer.

Finally, the 3-valued signal $\delta_{k}$ is transform into a 2-bits binary number $\nu_{k} \in\{00,01,10\}$, by the following operation.

$$
\nu_{k}=\left\{\begin{array}{lll}
00 & \text { if } & \delta_{k}=0 \\
01 & \text { if } & \delta_{k}=-1 \\
10 & \text { if } & \delta_{k}=1
\end{array}\right.
$$

The combination ' $11^{\prime}$ ' is not used in this process.

2) The model-based predictor: Has the role to estimate (reconstruct) the encoded signal $x_{k}$, namely $\hat{x}_{k}$, from the 2-bits binary signal $\nu_{k}$. It is composed of:

- The inverse of the level detector: $\varphi_{L D}^{-1}: \nu_{k} \mapsto \delta_{k} \mapsto \hat{\tilde{x}}_{k}$, which equations are:

$$
\delta_{k}=\left\{\begin{array}{rll}
0 & \text { if } & \nu_{k}=00 \\
-1 & \text { if } & \nu_{k}=01 \\
1 & \text { if } & \nu_{k}=10
\end{array}\right.
$$

and,

$$
\hat{\tilde{x}}_{k}=\hat{\tilde{x}}_{k-1}+\Delta \cdot \delta_{k}
$$

Due to the quantization, this map does not describe an "exact inverse operator" as it will be explained latter.

- The predictor. The model-based predictor, as its name indicated, uses the target closed-loop model as a basis for its design. The predictor is a dynamic operator mapping the "reconstructed" error $\hat{\tilde{x}}_{k}$ to the "reconstructed" state $\hat{x}_{k}$. Its structure is inspired by our previous works in [3], [5], and also in [6]. The predictor is a dynamic linear discrete-time operator that maps the output of the 
inverse level detector, to the signal prediction $\hat{x}_{k}$. Its structure depends upon the particular control used (state feedback or output feedback). For instance, for the RSTcontrol discussed here, it has the following form:

$$
\hat{x}_{k}=W\left[\frac{\gamma}{T} r_{k}+\hat{\tilde{x}}_{k}\right], \quad W \triangleq \frac{B R}{A_{c l}}
$$

Which results in the following error equation:

$$
\tilde{x}_{k}=W\left[\tilde{x}_{k}-\hat{\tilde{x}}_{k}\right]
$$

\section{B. Description of the variable length entropy coding VLE}

As mentioned in the introduction, high compression rates can only be reached by the use of entropy coding. By assigning some probability distribution to the events, the mean code length can be optimized. Run-length codes ${ }^{1}$, are a class of variable-length codes that are sub-optimal (when compared to the Huffman code), but have the advantage of avoiding buffering at the decoder side, and therefore reducing data transmission latency. An example used in [9] is described in Table I.

The VLE of length $N$ can be described as a memory map from $\nu_{k}$ to $\eta_{j}$. The VLE block contains a buffer of dimension $l$ that stores information of past values of $\nu_{k}$. The buffer dimension $l$ depends on $N$. The buffer information is used to build a run sequence resulting from the composition of $\nu_{k-l}, \ldots, \nu_{k}$. This sequence, which is a variable-length binary signal, is named the "run sequence" used to produce the output $\eta_{j}$. The variable-length nature of this sequence introduces a variable latency (asynchronous output) which is multiple of $T_{s}$. An example of a coding scheme of block

\begin{tabular}{|c|c|c|c|}
\hline \multicolumn{2}{|c|}{ Run sequence } & $\begin{array}{c}\text { Transmission period } \\
(\mathrm{sec})\end{array}$ & $\begin{array}{l}\text { Output } \eta_{j} \\
(N=3)\end{array}$ \\
\hline & 01 & $T_{s}$ & 000 \\
\hline & 10 & $T_{s}$ & 001 \\
\hline & 00 & $2 T_{s}$ & 010 \\
\hline & 00 & $2 T_{s}$ & 011 \\
\hline 00 & 00 & $3 T_{s}$ & 100 \\
\hline 00 & 00 & $3 T_{s}$ & 101 \\
\hline 00 & 00 & $3 T_{s}$ & 110 \\
\hline \multicolumn{2}{|r|}{ unused } & - & 111 \\
\hline
\end{tabular}
length $N=3$ is shown below.

TABLE I

RUN-LENGTH ENCODING.

Assuming that the coding sequence is independent and identically distributed, and that the upward crossing frequency equals the downwards crossing frequency, i.e.

$$
p=P(00), \quad P(01)=P(10)=\frac{1}{2}(1-p)
$$

where $p \in[0,1]$ is the probability to have an stand-still event. According to [9], the mean coding length, $C_{L}$, of this scheme is:

$$
C_{L}=2 \frac{1-p^{\left(2^{(N-1)}-1\right)}}{1-p} \text { bits }
$$

${ }^{1}$ Class of coding strategy that can decode information instantaneously. which has the limiting value: $\lim _{p \rightarrow 1} C_{L}=2^{N}-2$. The compaction ratio is $C_{R}=\frac{C_{L}}{N}$.

Let $R_{\nu}$ be the transmission rate when the 2-bits signal $\nu_{k}$ is sent synchronously at each $T_{s}$ without using an entropy code. This rate is given by $R_{\nu}=2\left[\right.$ bits $\left./ T_{s}\right]$.

When the VLE coding is used assuming a probability $p$ to the stand-still event, the mean transmission rate $R_{m}$ [bits $\left./ T_{s}\right]$ associated to a code of length $N$ is

$$
R_{m}=\frac{\text { number of bits sent }}{\text { mean transmission period }}=\frac{N}{T_{m}}
$$

where $T_{m}=\frac{C_{L}}{2}\left[T_{s}\right]$. This gives

$$
R_{m}=\frac{2 N}{C_{L}}=R_{\nu} \frac{N}{C_{L}}
$$

From this expression we can evaluate the potential improvement due to the entropy coding in terms of the mean rate needed for this scheme to work. In particular if $p$ is large enough so that $\frac{N}{C_{L}}<1$, then the VLE scheme will provide lowest rates. Table II compares these rates for different values of $N$ and $p$. Note that very low rates are required for large $N$ and probabilities close to one(the bit rate is reduced by an order of magnitude at $p=1$ ).

\begin{tabular}{|c|ccc|c|}
\hline$N$ & \multicolumn{3}{|c|}{$R_{m}$ (with VLE) } & $\begin{array}{c}R_{\nu} \\
\text { (without VLE) }\end{array}$ \\
\hline 3 & 3 & 1.7 & 1.00 & 2 \\
4 & 4 & 2.0 & 0.57 & 2 \\
5 & 5 & 2.5 & 0.33 & 2 \\
6 & 6 & 3.0 & 0.19 & 2 \\
\hline
\end{tabular}

TABLE II

MEAN TRANSMISSION RATE $R_{m}$ FOR $p=0, p=0.5$ AND $p=1$

\section{ERROR SYSTEM AND STABILITY CONDITIONS}

Following the assumptions maid in this paper (lossless channel transmission), we then have that $\nu_{k}=\hat{\nu}_{k}$, and that $\delta_{k}=\hat{\delta}_{k}$. In this case binary variables are not needed, and hence error equation can be described by real variables only.

\section{A. Error equations}

Introducing the following error definitions:

- $e_{k}=x_{k}-\frac{\gamma}{A_{c l}} r_{k}$ : the tracking error,

- $\tilde{x}_{k}=x_{k}-\hat{x}_{k}$ : the prediction error, and

- $\varepsilon_{k}=\tilde{x}_{k}-\hat{\tilde{x}}_{k}$ : error due to the non exact inverse mapping of the level detector, i.e. due to the map $\varphi_{L D} \circ \varphi_{L D}^{-1}$.

we have the closed-loop error system:

$$
\begin{aligned}
& e_{k}=W\left(q^{-1}\right) \tilde{x}_{k} \\
& \tilde{x}_{k}=W\left(q^{-1}\right) \varepsilon_{k}
\end{aligned}
$$

with $W=B R / A_{c l}$ being the stable operator defined previously. Note that the $\varepsilon_{k}=\varepsilon_{k}\left(\tilde{x}_{k}\right)$, and thereby the above error equation can be seen as two systems in cascade, i.e. the output of the autonomous system (6) is the input of the stable system (5). For stability purposes it is thus sufficient to demonstrate the stability properties of the sub-system (6). 
Note that $\varepsilon_{k}$ writes as:

$$
\begin{aligned}
\varepsilon_{k} & =\tilde{x}_{k}-\hat{\tilde{x}}_{k} \\
& =\tilde{x}_{k}-\varphi_{L D} \circ \varphi_{L D}^{-1}\left\{\tilde{x}_{k}\right\} \\
& =\tilde{x}_{k}-\tilde{\varphi}_{L D}\left\{\tilde{x}_{k}\right\}
\end{aligned}
$$

where $\tilde{\varphi}_{L D} \triangleq \varphi_{L D} \circ \varphi_{L D}^{-1}: \tilde{x}_{k} \mapsto \hat{\tilde{x}}_{k}$. Note that this map is dynamic, defined by the following relation:

$$
\hat{\tilde{x}}_{k}=\hat{\tilde{x}}_{k-1}+\Delta \cdot \delta_{k}
$$

with $\delta_{k}=f\left(\tilde{x}_{k}\right)$ as defined before. The sub-system (6)-(7) can be then seen as a feedback system,i.e.

$$
\begin{aligned}
\tilde{x}_{k} & =W\left(q^{-1}\right) \varepsilon_{k} \\
& =W\left(q^{-1}\right)\left(\tilde{x}_{k}-\hat{\tilde{x}}_{k}\right) \\
& =W\left(q^{-1}\right)\left(\tilde{x}_{k}-\frac{\Delta}{1-q^{-1}} \delta_{k}\left(\tilde{x}_{k}\right)\right)
\end{aligned}
$$

Ideally we would like that the map $\tilde{\varphi}_{L D}$ be a linear map with unitary gain. This ideal goal is hampered by several factors:

- unknown initial conditions of $\tilde{x}_{0}$,

- badly chosen $T_{s}$, and $\Delta$, and

- chattering in the neighborhood of the quantum $\Delta$.

In particular, large sampling times $T_{s}$, and too small quantum $\Delta$ may results in signal variation of more than one level, which may leads to unrecovered bias in the estimated, leading to potential instabilities for unstable open-loop systems. This stabilities issues are analyzed next for a system of one dimension.

\section{B. Stability properties}

Consider the stabilization problem $(r=0)$ of the following simple unstable system $\frac{B\left(q^{-1}\right)}{A\left(q^{-1}\right)}=\frac{b q^{-1}}{1-a q^{-1}}$, with $2>|a|>1$, and the control law $u=k x_{k}$. Let $1>a_{c}>0$ be the desired closed loop poles, the required gain to reach such closed-loop specification is $k=\left(a-a_{c}\right) / b$. This particular choice leads to the error equations (5)-(6) with $W\left(q^{-1}\right)=\frac{\left(a-a_{c}\right) q^{-1}}{1-a_{c} q^{-1}}$. Due to the cascade structure of such error equation arrangement, we mainly will concentrate in the equation (6) which captures most of the difficulties. To this aim we will concentrate on the following set of equations, which describes the error feedback interconnection.

$$
\begin{aligned}
\tilde{x}_{k+1} & =a_{c} \tilde{x}_{k}+\left(a-a_{c}\right) \varepsilon_{k}, \quad \varepsilon_{k}=\tilde{x}_{k}-\hat{\tilde{x}}_{k} \\
\hat{\tilde{x}}_{k} & =\hat{\tilde{x}}_{k-1}+\Delta \operatorname{sign}\left(l_{k}-l_{k-1}\right), \quad l_{k}=\left\lfloor\frac{\tilde{x}_{k}}{\Delta}-\frac{1}{2}\right\rfloor
\end{aligned}
$$

The analysis is divided into two steps:

- Rate level condition. We first derive conditions on $a$, and a domain $B_{\rho_{1}}$ for $\tilde{x}_{k}$ that ensures that no more than one level change can be effectuated, i.e. $\left|l_{k}-l_{k-1}\right| \leqslant 1$,

- Invariance condition. Then, by a Lyapunov-like analysis we show that this domain is indeed an invariant; solutions $\tilde{x}_{k}$ starting in $B_{\rho_{1}}$ do not leave this domain.

\section{Rate level condition}

We seek here to establish condition on $\left|\tilde{x}_{k}\right|, \forall k \in \mathbb{Z}^{+}$such that the rate change in the level detector be at most one. To be consistent with this aim, we need to assume in the sequel that the encoder/deconder internal states are suitable initialized. That is, $\hat{x}_{0}$, and $l_{0}$ are such that: $\varepsilon_{0}<\Delta / 2$, and $\hat{\tilde{x}}_{0}=\Delta l_{0}$ at $k=0$.

Lemma 1: Consider unstable systems limited by the relation $a<2+a_{c}<3$, and let define the compact set, $B_{\rho_{1}}$, as:

$$
B_{\rho_{1}}=\left\{\tilde{x}_{k}:\left|\tilde{x}_{k}\right|<\rho_{1}\right\}, \quad \rho_{1}=\frac{\left(1-\frac{\left(a-a_{c}\right)}{2}\right)}{1-a_{c}} \Delta
$$

with $\rho_{1}>0$. Then for all $\left|\tilde{x}_{k}\right| \in B_{\rho_{1}}$ the following holds, $\forall k \in \mathbb{Z}^{+}$:

i) $\left|\tilde{x}_{k}-\tilde{x}_{k-1}\right|<\Delta$,

furthermore, $i$ ) implies the following two equivalent inequalities:

ii) $\left|l_{k}-l_{k-1}\right| \leqslant 1$

iii) $\left|\varepsilon_{k}\right| \leqslant \Delta / 2$

Proof: Let us start with the last part of this result, i.e. $(i)$ $\Rightarrow\{(i i) \Leftrightarrow(i i i)\}$. By inspection, it is easy to see that $(i) \Rightarrow$ (ii); if the rate of change of $\tilde{x}_{k}$ is strictly smaller than $\Delta$ then the level change is limited, by definition, to a maximum one. In turn, $(i i) \Rightarrow(i i i)$, results from the following arguments.

If the initialization condition $\hat{\tilde{x}}_{0}=\Delta l_{0}$ and $(i i)$ holds, then we have that $\hat{\tilde{x}}_{k}=\Delta l_{k}, \forall k \in \mathbb{Z}^{+}$. Then it follows that the error between the true estimation error, and the reconstructed one is bounded by the amount $\Delta / 2$, i.e.

$$
\begin{aligned}
\varepsilon_{k} & =\tilde{x}_{k}-\hat{\tilde{x}}_{k}=\tilde{x}_{k}-\Delta l_{k}=\tilde{x}_{k}-\left\lfloor\frac{\tilde{x}_{k}}{\Delta}-\frac{1}{2}\right\rfloor \\
& =\Delta\left(\frac{\tilde{x}_{k}}{\Delta}-\left\lfloor\frac{\tilde{x}_{k}}{\Delta}-\frac{1}{2}\right\rfloor\right) \leqslant \frac{\Delta}{2} .
\end{aligned}
$$

Inversely, if ( $i i i)$ holds, it is easy by inspection to see that (ii) is true.

Now the first part of the lemma $(i)$ is proven. From (8) we have

$$
\left|\tilde{x}_{k+1}-\tilde{x}_{k}\right|<\left(1-a_{c}\right)\left|\tilde{x}_{k}\right|+\left(a-a_{c}\right)\left|\varepsilon_{k}\right|
$$

assume for the moment that (iii) holds, then condition for fulfilling $i$ ) is that

$$
\left|\tilde{x}_{k+1}-\tilde{x}_{k}\right|<\left(1-a_{c}\right)\left|\tilde{x}_{k}\right|+\left(a-a_{c}\right) \mid \Delta / 2<\Delta
$$

or equivalently that

$$
\left|\tilde{x}_{k}\right|<\frac{\left(1-\frac{\left(a-a_{c}\right)}{2}\right)}{1-a_{c}} \Delta=\rho_{1}
$$

For this expression to be valid (i.e. $\rho_{1}>0$ ), we require the condition $a<2+a_{c}<3$. To conclude, assume that (10) holds independently to $(i)-(i i i)$ (as it will be shown latter in the next section). Now if $(i)$ holds we just show that this implies (iii), which in turn and together with (10), implies (i). 


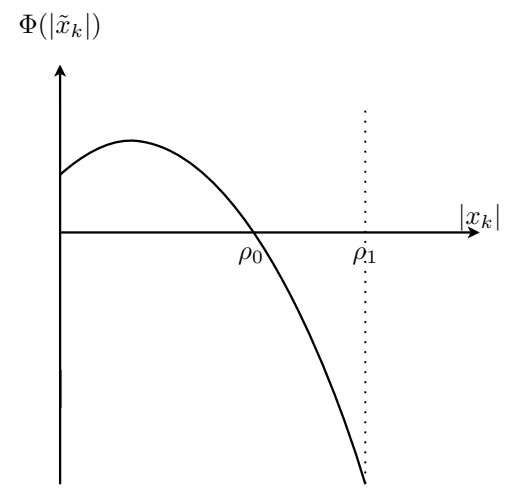

Fig. 3. $\Phi\left(\left|\tilde{x}_{k}\right|\right)$.

\section{Invariance condition}

Question here is to find under which conditions we can ensure the invariance of the set $B_{\rho_{1}}$. This invariance condition is clearly needed to preserve the rate level condition mentioned previously. We assume initially that the observation error is inside that set, and we look for the condition such that this signal does not leaves $B_{\rho_{1}}$.

To this aim, consider the Lyapunov function $V_{k}=\tilde{x}_{k}^{2}$, and its rate variation $\nabla V_{k} \triangleq \tilde{x}_{k+1}^{2}-\tilde{x}_{k}^{2}$, i.e.

$$
\begin{aligned}
\nabla V_{k} & =\left(a_{c}^{2}-1\right)\left|\tilde{x}_{k}\right|^{2}+2\left(a-a_{c}\right) a_{c} \varepsilon_{k} \tilde{x}_{k}+\left(a-a_{c}\right)^{2} \varepsilon_{k}^{2} \\
& \leqslant\left(a_{c}^{2}-1\right)\left|\tilde{x}_{k}\right|^{2}+\left(a-a_{c}\right) a_{c}\left|\tilde{x}_{k}\right| \Delta+\frac{\left(a-a_{c}\right)^{2}}{4} \Delta^{2} \\
& \triangleq \Phi\left(\left|\tilde{x}_{k}\right|\right)
\end{aligned}
$$

Where the last inequality is results from the hypothesis that initially we assume $\tilde{x}_{k} \in B_{\rho_{1}}$, or equivalently (see Lemma 1) that $\left|\varepsilon_{k}\right| \leqslant \Delta / 2$.

The shape of the polynomial $\Phi\left(\left|\tilde{x}_{k}\right|\right)$ is shown in Figure 3, this function has two roots, one negative and other positive. The positive root, is $\rho_{0}$, and is given by

$$
\rho_{0}=\frac{\left(a-a_{c}\right)}{2\left(1-a_{c}\right)} \Delta
$$

It is necessary for stability that $\rho_{0}<\rho_{1}$, else a local stability region may not exist. It is easy to show that this condition is valid as long as $a<a_{c}+1$, which is the same condition already assumed by Lemma 1 .

The value of $\rho_{0}$ defines the limit (somewhat conservative) after which the function $V_{k}$, and hence the norm of $x_{k}$ decrease. Below that limit the function may grow. The worst case growth in the interval $\left|\tilde{x}_{k}\right| \in\left[0, \rho_{0}\right]$, can be estimated from the relation

$$
\tilde{x}_{k+1}^{2} \leqslant\left(a_{c}\left|\tilde{x}_{k}\right|+\frac{\left(a-a_{c}\right)}{2} \Delta\right)^{2} \triangleq \psi\left(\left|\tilde{x}_{k}\right|\right)
$$

As the function $\psi\left(\left|\tilde{x}_{k}\right|\right)$ is convex in $\left|\tilde{x}_{k}\right| \in\left[0, \rho_{0}\right]$ its maximum is located at the extremes of this interval. The worst case growth is then defined by the following relation:

$$
x_{\max }=\max \left\{\sqrt{\psi(0)}, \sqrt{\psi\left(\rho_{0}\right)}\right\}=\Delta \frac{\left(a-a_{c}\right)}{2\left(1-a_{c}\right)}
$$

Finally, the set $B_{\rho_{1}}$ is invariant if $x_{\max }<\rho_{1}$, i.e.

$$
\Delta \frac{\left(a-a_{c}\right)}{2\left(1-a_{c}\right)}<\Delta \frac{\left(1-\frac{\left(a-a_{c}\right)}{2}\right)}{1-a_{c}}
$$

working out details of this inequality, it can be shown that this equality holds if $a-a_{c}<1$, for all $a_{c} \in(0,1)$. Note that this is a stronger condition than the one in Lemma 1 as it is derived from a more conservative analysis.

The following theorem summarizes the main result.

Theorem 1: Assume that the coding algorithm is initialized such that $\hat{x}_{0}$, and $l_{0}$ are such that: $\varepsilon_{0}<\Delta / 2$, and $\hat{\tilde{x}}_{0}=\Delta l_{0}$. Consider system satisfying $a-a_{c}<1$, with initial condition in the set $\tilde{x}_{0} \in B_{\rho_{1}}$. Then:

- $\tilde{x}_{k} \in B_{\rho_{1}}, \forall k \in \mathbb{Z}^{+}$,

- $\exists k_{0}:\left|\tilde{x}_{k}\right| \leqslant \rho_{0}, \forall k \geqslant k_{0}$, and

- $\lim _{k \rightarrow \infty} d\left(x_{k}, \mathcal{B}_{\beta}\right)=0$.

where $d\left(x_{k}, \mathcal{B}_{\beta}\right)$ is the minimum Euclidean distance from $x_{k}$ to any point within the ball

$$
\mathcal{B}_{\beta}:=\{x \in \mathbb{R}:\|x\|<\beta\},
$$

and $\beta$ is a constant that depends on $\rho_{0}$, and on the infinite norm of $W\left(q^{-1}\right)$.

Proof: The first two statements follow from the previous analysis, the last statement result from equation (5), i.e; $\left|x_{k}\right| \leqslant|| W|| \cdot\left|\tilde{x}_{k}\right|$. Details for the derivation of this property are similar to the ones used in [3], and [5].

\section{Simulation evaluation}

We consider the following simple system

$$
\frac{B\left(q^{-1}\right)}{A\left(q^{-1}\right)}=\frac{b q^{-1}}{1-a q^{-1}}
$$

The controller is: $u_{k}=-k \hat{x}_{k}+\gamma r_{k}$ obtained from the closedloop specification given by $A_{c l}=\left(1-a_{c l} q^{-1}\right), k=\frac{a-a_{c l}}{b}$ and $\gamma=1-a_{c}$. Parameter used in simulations are: $a=1.1$, $b=1, a_{c}=0.9, T_{s}=0.05(\mathrm{sec}), \Delta=0.02, x_{0}=0$ and $\hat{x}_{0}=-0.01$ so $\tilde{x}_{0}=0.01$.

The purpose of this section is to evaluate in simulation the proposed algorithm and to discuss several issues concerning the algorithm implementation. Among these, we have: initial synchronization, effect of the quantum $\Delta$ and the sampling time $T_{s}$, among others.

\section{A. Initial conditions}

Initial conditions of the predictor at the encoder side $\hat{x}_{0}$ need to be synchronized with initial condition predictor at the decoder side. This requires a specific initialization procedure that send this initial information before the coding process is triggered. 


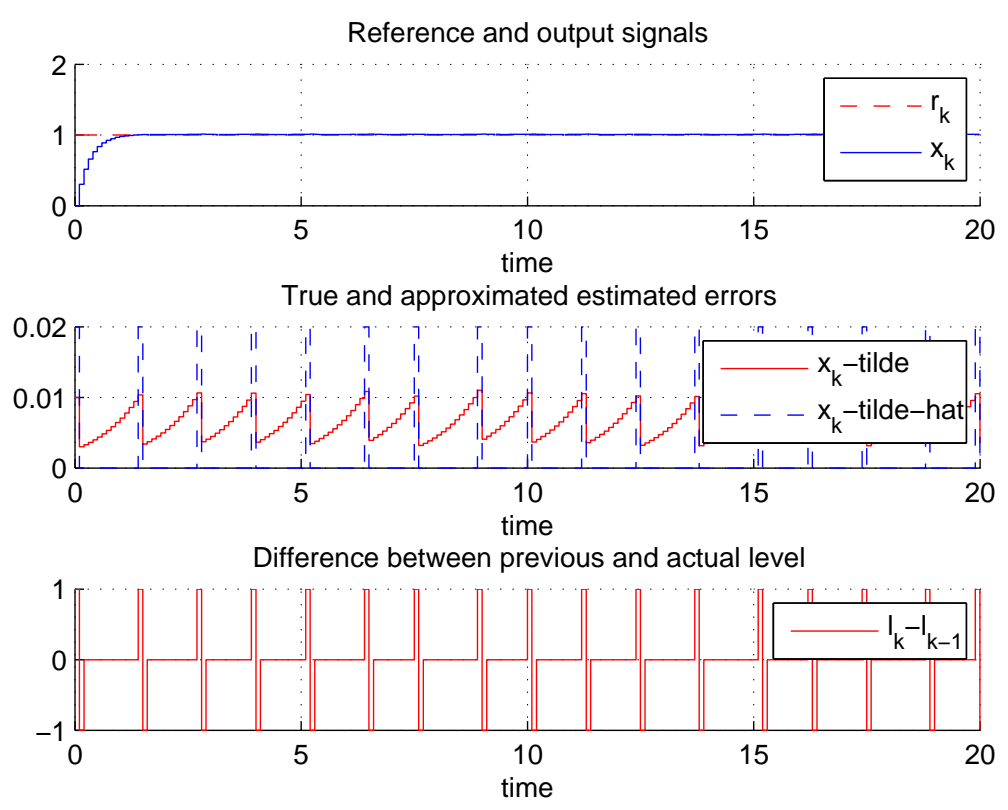

Fig. 4. Simulation results with $\Delta=0.02 ; T_{s}=0.05$ yielding $\left|l_{k}-l_{k-1}\right| \leqslant 1$. Output and reference (upper), $\tilde{x}$ vs. $\hat{\tilde{x}}$ (middle), and $l_{k}-l_{k-1}$ (lower)

\section{B. Quantum $\Delta$ and sampling time $T_{s}$}

The value of $\Delta$ has an important impact on the quality of the estimates and the system stability. Larger values of $\Delta$ will enlarge the attraction domain, but it will degrade the estimated quality. Inversely, small values for $\Delta$ will improve the reconstructed signal quality, but it will diminish the local attraction domain.

The impact of $T_{s}$ can be seen from the necessary condition for stabilization, i.e. $a=e^{\alpha T_{s}}<2$, where $\alpha$ is the open-loop pole of the continuous system. For unstable systems, $T_{s}$ is limited by the relation $T_{s}<\ln (2) / \alpha$. The more the system is unstable the smaller need to be $T_{s}$ and hence the transmission bit rates need to be increased.

The Figure 4, shows a simulation where parameters are selected to satisfy the stability conditions. As a consequence, the change of level is limited to one.

\section{Event distribution and its impact in the mean transmission rate}

Note that practically all parameters of the control scheme affects the spectrum of the evolution of $\delta_{k}$, and in particular the $\Delta$, and $T_{s}$, but also the magnitude of open-loop unstable poles. Figure 5 shows the resulting histogram of $\delta_{k}$ for two simulations with two different values of $a$.

The results show that for small values of $a$ the frequency spectrum of $\delta_{k}$ is reduced, and hence the event $\delta_{k}=0$ has higher probability to occur. We recall that distributions with high roll-off will be benefic for data compression, as illustrated by the VLE algorithm. We can then conclude that open-loop instable systems with a high degree of instability are less adapted for entropy coding. The resulting compression ratio are reported in Table III. Higher compression rates
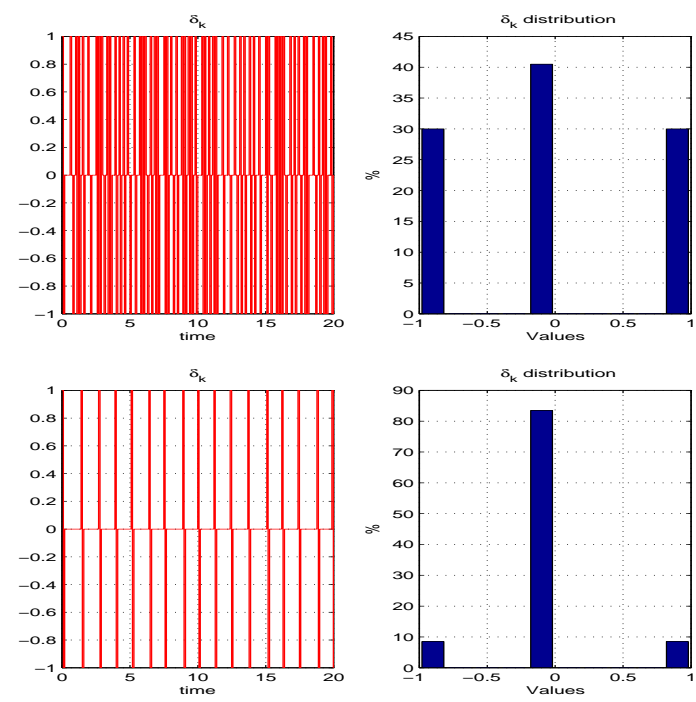

Fig. 5. Simulations with $\Delta=0.02 ; T_{s}=0.05 ; a=1.6$ (upper), $\Delta=0.02 ; T_{s}=0.05 ; a=1.1$ (bottom), Time evolution of $\delta_{k}$ (left), histogram of events (right)

are thus obtained for the cases where $p$ is higher, i.e. the bottom figures in Fig.5.

From the Table III we can see that for $a=1.1$, the best choice is a VLE coding of length $N=4$, or $N=5$, whereas for the system with $a=1.6$ only the VLE with $N=3$ improves over the one without entropy coding.

\section{CONCLUSIONS}

In this paper we have investigated the possibility to use entropy coding in the context of networked controlled sys- 


\begin{tabular}{|c|cc|c|}
\hline$N$ & \multicolumn{2}{|c|}{$R_{m}$ (with VLE) } & $R_{\nu}$ \\
& $p=0.4 ;$ & $p=0.8$ & (without VLE) \\
& $a=1.6 ;$ & $a=1.1$ & \\
\hline 3 & 1.92 & 1.23 & 2 \\
4 & 2.40 & 1.01 & 2 \\
5 & 3.00 & 1.03 & 2 \\
6 & 3.60 & 1.20 & 2 \\
\hline
\end{tabular}

TABLE III

MEAN TRANSMISSION RATES FROM DATA SHOWN IN FIG. 5.

tems. The main motivation has been to explore the benefits in terms of mean transmission rate. In particular we have analyzed the case of variable-length encoder VLE, which in spite of its sub-optimality do not require buffering at the decoder side, and hence reduce latency.

We have shown that the scheme results in a local stable system with an attraction domain and a final precision depending on the value of the level granularity $\Delta$. Finally we have demonstrated by simulation the benefits that can be reached by using this type of coding, and for a given system with a fixed scenario, how to select the length of the VLE code. From this study it results also that system with a low degree of instability (eigenvalues closed to one), the optimal value of $N$ is in general larger than for system with high degree of instability.

\section{REFERENCES}

[1] Brockett R.-W. and Liberzon D. Quantized feedback stabilization of linear systems. IEEE Transactions on Automatic Control, 45(7):12791289, July 2000.

[2] C. Canudas-de-Wit, J.Jaglin, and C Siclet. Energy-aware 3-level coding and control co-design for sensor network systems. To appear in Conference on Control Application, 2007.

[3] C. Canudas-de-Wit, F. Rubio, J. Fornes, and F. Gomez-Estern. Differential coding in networked controlled linear systems. American Control Conference. Silver Anniversary ACC. Minneapolis, Minnesota USA, June 2006.

[4] N. Elia and S.-K. Mitter. Stabilization of linear systems with limited information. IEEE Transaction on Automatic Control, 46(9):13841400, September 2001.

[5] F. Rubio F. Gomez-Estern, C. Canudas-de-Wit and J. Fornes. Adaptive delta-modulation coding in networked controlled systems. Submitted to American Control Conference, New York, USA USA, June 2007.

[6] Hespanha J.-P., Ortega A., and Vasudevan L. Towards the control of linear systems with minimum bit-rate. In 15th Int. Symp. Mathematical Theory of Networks and Systems (MTNS), Notre Dame, IL, USA, 2002.

[7] C.T. Abdallah I. Lopez and C. Canudas-de-Wit. Compensation schemes for a delta-modulation-based ncs. Submitted to ECC'07 USA, 2007.

[8] H. Ishii and T. Başar. Remote control of lti systems over networks with state quatization. System and Control Letters, (54):15-31, 2005.

[9] J.W. Mark, and Tood, T.D. A nonuniform sampling approach to data compression. IEEE Transaction on Communications, 29(1):24-32, January 1981.

[10] K. Li and J. Baillieul. Robust quatization for diginal finite communication bandwidth (dfcb) control. IEEE Transaction on Automatic Control, 49(9):1573-1584, September 2004.

[11] D. Liberzon. On stabilization of linear systems with limited information. IEEE Transaction on Automatic Control, 48(2):304-307, February 2003.

[12] Lemmon M. and Q. Ling. Control system performance under dynamic quatization: the scalar case. In 43rd IEEE Conference on Decision and Control, pages 1884-1888, Atlantis, Paradice Island, Bahamas, 2004.

[13] J.G. Proakis. Digital Communications. McGraw-Hill, Inc. Series in electrical and computer enginering, 2001.
[14] S. Tan, Xi Wei, and J.-S. Baras. Numerical study of joint quatization and control under block-coding. In 43rd IEEE Conference on Decision and Control, pages 4515-4520, Atlantis, Paradice Island, Bahamas, 2004. 